Recepción: 28 / 12 / 2017

Aceptación: 20 / 02 / 2018

Publicación: 08 / 05 / 2018
Ciencias económicas y empresariales

Artículo de Revisión

\title{
Administración del talento humano, calidad de vida laboral y su doble dimensión: objetiva y subjetiva
}

\author{
Administration of human talent, quality of working life and its double \\ dimension: objective and subjective
}

\section{Administração do talento humano, qualidade de vida no trabalho e sua dupla dimensão: objetivo e subjetivo}

\author{
Oscar A. Gómez-Cabrera ${ }^{I}$ \\ ogomezcabrera@yahoo.es \\ Rocío del P. Aguilar-Salazar II \\ aguilarsalazarrocio@gmail.com
}

Correspondencia: ogomezcabrera@yahoo.es

\begin{abstract}
${ }^{I}$ Magister en Docencia Universitaria en el Sistema Modular, Magister en Educación a Distancia, Ingeniero Comercial, Licenciado en Administración de Empresas, Docente de la Universidad Nacional de Loja, Loja Ecuador.

II Magister en Educación a Distancia, Master en Dirección y Gestión Turística, Licenciada en Administración de Empresas Turísticas, Tecnóloga en Administración de Empresas Turísticas, Docente de la Universidad Nacional de Loja, Loja Ecuador.
\end{abstract}




\section{Resumen}

La doble dimensión que la calidad de vida laboral tiene, se compone de los factores involucrados en la relación laboral (hombre - ambiente de trabajo) en el ámbito de la empresa. La Calidad de Vida Laboral debe satisfacer las expectativas del trabajador y por ende las de la organización, sin subestimar una sobre la otra. Es fundamental para las empresas y organizaciones identificar las necesidades de sus trabajadores (humanas, psicológicas, sociales, culturales, contractuales, salariales, seguridad e higiene y autorrealización), y orientarse al diseño de infraestructuras, políticas, objetivos, metas y planes organizacionales, que garanticen la satisfacción y bienestar de sus trabajadores y en consecuencia el cumplimiento de la visión y misión de la organización. Surge en este contexto de la administración de empresas y en particular de su talento humano la justificación del presente artículo, que pretende abordar una reflexión sobre las dimensiones que posee la calidad de vida laboral. El objetivo general del presente estudio consistió en analizar la dimensión objetiva y subjetiva de la calidad de vida laboral y sus relaciones para mejorar las dimensiones y criterios durante el análisis de los factores presentes en los programas de calidad de vida laboral de las empresas y organizaciones.

Palabras clave: empresas y organizaciones; administración del talento humano; calidad de vida laboral subjetiva; calidad de vida laboral objetiva.

\section{Abstract}

The double dimension that the quality of working life has, is made up of the factors involved in the labor relationship (man - work environment) in the field of the company. The Quality of Work Life must meet the expectations of the worker and therefore those of the organization, without underestimating one over the other. It is fundamental for companies and organizations to identify the needs of their workers (human, psychological, social, cultural, contractual, salary, safety and hygiene and self-realization), and to be oriented to the design of infrastructures, policies, objectives, goals and organizational plans, which guarantee the satisfaction and wellbeing of its workers and consequently the fulfillment of the vision and mission of the organization. The justification of this article arises in this context of the administration of companies and in particular of its human talent, that tries to approach a reflection on the dimensions that have the quality of labor life. The general objective of this study was to analyze the objective and subjective dimension of the quality of working life and its relationships to 
improve the dimensions and criteria during the analysis of the factors present in the quality of work life programs of companies and organizations.

Keywords: companies and organizations; human talent management; subjective quality of work life; objective quality of working life.

\section{Resumo}

A dupla dimensão que a qualidade da vida profissional tem, é composto dos fatores envolvidos na relação de emprego (homem - ambiente de trabalho) no campo dos negócios. Qualidade de Vida no Trabalho deve atender as expectativas do trabalhador e, portanto, a organização, sem subestimar um sobre o outro. É essencial para as empresas e organizações para identificar as necessidades de seus trabalhadores (humano, psicológico, social, cultural, contrato, salário, saúde e segurança e auto-realização) e orientar o projeto de infra-estrutura, políticas, objetivos, metas e planos organizacionais, que garantir a satisfação e o bem-estar de seus trabalhadores e, consequentemente, o cumprimento da visão e missão da organização. Ela surge no contexto de administração de empresas e em particular a sua justificação talento humano deste artigo, que visa abordar uma reflexão sobre as dimensões que tem a qualidade de vida no trabalho. $\mathrm{O}$ objetivo geral deste estudo foi analisar a dimensão objetiva e subjetiva da qualidade de vida no trabalho e seus relacionamentos para aumentar o tamanho e os critérios para a análise dos fatores em programas de qualidade de vida das empresas e organizações a trabalhar.

Palavras chave: empresas e organizações; gestão de talentos humanos; qualidade subjetiva da vida profissional; qualidade objetiva da vida profissional.

\section{Introducción}

La Administración del talento humano es una especialidad de la administración de empresas que se encarga de brindar estrategias para el desarrollo profesional, que estén alineadas a la misión y visión organizacional para dar cumplimiento al logro de los objetivos corporativos (López P y col, 2017). En ese sentido, según Matabajoy-Montilla y col (2018), una de las funciones principales de la administración del talento humano es desarrollar procesos articulados que generen desarrollo humano y organizacional, que se logra a través de los procesos de administración (selección, contratación, inducción) y desarrollo del talento humano 
(capacitación, calidad de vida laboral, salud laboral, clima organizacional y evaluación de desempeño).

Los trabajadores en una organización desempeñan un papel fundamental en el cumplimiento de los objetivos corporativos, en ese sentido, es importante identificar los procesos de desarrollo de talento humano, entre los cuales destaca la Calidad de Vida Laboral (CVL).

En base al criterio de que las personas pasan la mayor parte de su tiempo viviendo o trabajando en organizaciones. Las personas nacen, crecen, se educan, trabajan y se divierten dentro de organizaciones, las cuales determinan que los individuos dependan cada vez más de las actividades en grupos laborales (Chiavenato, 1999).

En este sentido, las sociedades están organizadas con base a las principales necesidades de sus miembros, sin embrago, estas necesidades ya no están limitadas, a la alimentación, vestidos, fisiológicas, etc.

Actualmente, las personas pretenden ir más allá de las necesidades básicas, por su naturaleza el hombre busca estar y sentirse bien en los ambientes en los cuales se desarrolle, su sentido de responsabilidad lo invita a proteger sus seres queridos, su entorno familiar y su trabajo, en tal sentido, como todo trabajador en general busca la mejor alternativa que le permita, además de satisfacer sus diferentes necesidades evolucionar y desarrollar sus talentos, así mismo, las organizaciones buscan trabajadores que contribuyan a maximizar sus ganancias y hacer de la empresa la más exitosa y competitiva del mercado.

En conformidad con la antes expuesto, muchos directivos y dueños de empresas han entendido la importancia que tiene la generación de estrategias que den como resultado la satisfacción del trabajador y el éxito organizacional. Una de las estrategias que ha tomado vigor en las últimas décadas es el de Calidad de Vida Laboral o CVT (Calidad de Vida en el Trabajo), ésta debe considerar los principales factores componentes de la relación laboral, en tal sentido, la calidad de vida laboral abarca dos dimensiones complementarias, que abarcan al hombre como un todo, es decir, que considera todas las necesidades y expectativas que tiene como individuo, como integrante de un equipo de trabajo y como parte de una organización, en decir, la CVT, abarca una dimensión objetiva y una subjetiva (Blanch y col, 2003). 
La presente disertación tiene por objetivo analizar el constructo de la Calidad de Vida Laboral y su doble dimensión, objetiva y subjetiva, a través de una investigación bibliografía.

\section{Desarrollo}

\section{Calidad de vida laboral}

El tema de la CVL (Calidad de Vida Laboral) ó CVT (Calidad de Vida en el Trabajo), se puede considerar de reciente interés, puesto que toma su auge en la década de los años 70 y alcanza los conceptos básicos más concisos en 1983, así lo señalan (Jacobs y Homburguer, 1992).

Este tema toma su fuerza dentro de las empresas de EEUU, debido a la necesidad de mejorar la calidad de los resultados, siendo esta la grande ventaja que tenían las compañas asiáticas. Ante esta imperante necesidad, estas empresas norteamericanas aplicaron programas de calidad de vida laboral, y los resultados fueron muy exitosos, una de las primeras en aplicar este tipo de programas fue General Motors, luego se le unió Ford obteniendo buenos resultados, como lo refiere (Garzón, 2005).

Muchos autores han utilizado el término de Calidad de Vida Laboral, luego de analizar varios de estos autores, Garzón (2005), ha definido la Calidad de Vida Laboral como una diferente forma de vida dentro de la organización, que busca el desarrollo del trabajo, así como la eficiencia empresarial. También señala Garzón (2005), que adoptar e implementar proyectos de calidad de vida en el trabajo traerá muchos beneficios tanto para la empresa como para el trabajador, pudiéndose reflejar en:

- Evolución y desarrollo del trabajador.

- Una elevada motivación.

- Mejor desenvolvimiento de sus funciones.

- Menor rotación en el empleo.

- Menos tasas de ausentismo.

- Menos quejas.

- Tiempo de ocio reducido.

- Mayor satisfacción en el empleo.

- Mayor eficiencia en la organización. 
Sin embargo, Garzón (2005), también expone que no siempre los proyectos de calidad de vida en el trabajo se traducen en beneficios esperados y menciona algunas condiciones adversas para el logro de los resultados esperados, los cuales se mencionan a continuación:

a. Esto se da en las organizaciones en las que los empleados son incapaces de asumir una nueva responsabilidad, son reacios a trabajar en grupo, les desagrada reaprender, les desagradan los deberes más complejos, por lo que implantar proyectos de CVT se hace muy difícil.

b. Los mejores trabajos no concuerdan con los pagos que se reciben.

c. Al mejorar los empleos, los empleados ahora piensan que lo que reciben de salario no va de acuerdo con los nuevos deberes o los nuevos procedimientos de trabajo, por lo que desean obtener un mejor sueldo.

d. Aumento de costos.

e. El equipo tecnológico no es adaptable.

Las organizaciones deben desarrollar programas de CVT ó CVL, y así alcanzar un mayor y mejor compromiso de los directivos y de los trabajadores. La calidad de vida laboral, definida por Blanch y col (2003), expone que por calidad de vida laboral se entiende el grado de satisfacción y bienestar físico, psicológico y social experimentado por las personas en su puesto y en su entorno de trabajo. Abarca una doble dimensión:

- Objetiva: la calidad de vida laboral está relacionada con las condiciones de trabajo (ecológicas, materiales, técnicas, contractuales, salariales, de seguridad e higiene, de protección social, de estabilidad en empleo, de estructura organizacional, de diseño de puesto, etc.)

- Subjetiva: asociada a las percepciones y valores sobre la mejorabilidad de las condiciones laborales, sobre el stress laboral experimentado, sobre el ambiente social (tanto a lo concerniente a las relaciones horizontales con compañeros de trabajo como en las verticales, del trato recibido de la dirección), sobre el clima organizacional, sobre la estructura percibida 
de oportunidades de promoción, sobre el grado en que se siente que el trabajo contribuye al propio desarrollo personal, etc.

\section{Dimensión Objetiva de la calidad de vida laboral}

Hace referencia a las condiciones medibles del entorno que en sí mismas ayudan a que el trabajador tenga una vida laboral mejor, el concepto se identifica con los indicadores asociados a las condiciones externas basadas en el contexto donde labora el trabajador (Casas y col, 2002), se mencionan los siguientes aspectos y condiciones objetivas del entorno laboral:

- Medioambiente físico.

- Condiciones de confort y funcionalidad.

- Seguridad (higiene, manejo de productos tóxicos o peligrosos, contagios)

- Medioambiente tecnológico.

- Adecuación de equipos e instrumentos.

- Mantenimiento y suministros.

- Medioambiente contractual.

- Referido al salario (objetivo y subjetivo)

- Estabilidad laboral (explorada en carátula)

- Cobertura jurídica.

- Medioambiente productivo.

- Horarios

- Recursos

- Adecuación de medios humanos (sobrecarga cuantitativa)

- Adecuación demandas (sobrecarga cualitativa: - Positiva: Demanda competencias excesivas. - Negativa: Infrautilización)

- Medioambiente profesional.

- Promoción y carrera profesional 
- Capacitación e investigación

Cabe resaltar que la calidad de vida tiene importante repercusión social al ser uno de los responsables de los cambios en las actitudes y formas de pensar de los profesionales y gestores de las organizaciones, y de la relevancia que hoy cobra la persona como centro a partir de la cual se diseña cualquier tipo de intervención o intento de mejora en sus condiciones de vida.

Se ha pasado de un modelo de organización tradicional que consideraba la prestación de recursos y servicios como un fin en sí mismos, a una organización que Schalock y col. (2007) es definida como un "puente hacia la comunidad", en la que la finalidad de ésta no sólo es promover y ofertar servicios, sino utilizarlos para generar resultados personales positivos, como lo refiere (Cuesta, 2009)

Como ha sido expuesto, el concepto de CVL es multidimensional y está relacionado con los aspectos más relevantes para la satisfacción, la motivación y el rendimiento laboral de los trabajadores. La identificación de esta dimensión, siendo muy relevante para la CVL en los profesionales, hará posible determinar los aspectos que deben ser mejorados en las organizaciones, modelar y mejorar los problemas identificados, medir resultados, así como realizar comparaciones, aunando los objetivos de los trabajadores y de la organización, como lo señalan Casas y col (2002).

En este sentido, es conveniente resaltar que para garantizar CVL al trabajador y por ende proveer satisfacción laboral al mismo, es imperante controlar el Medioambiente Físico, es decir, identificar los riesgos del entorno laboral y las actividades propias del puesto de trabajo, con el fin de eliminar o mitigar los límites de tolerancia de los riegos ya que éstos pueden originar enfermedades y accidentes ocupacionales. Casas y col (2002), refieren que se puede encontrar todo tipo de riesgos: físicos (radiaciones, ruidos, etc.), químicos (gases anestésicos, etc.), biológicos (infecciones víricas y bacterianas), disergonómicos relacionados con la fatiga física (riesgos posturales, cargas excesivas, etc.) y psicosociales. Todos estos factores a los que además se puede añadir la falta de espacio físico o la inadecuación del mismo, etc., inciden en la CVL percibida por los trabajadores y pueden llegar a ser un estresor importante en el trabajo.

Además, es preciso tener en cuenta que, en ocasiones, la exposición en el lugar de trabajo a factores ambientales no deseables se da a dosis relativamente bajas, que sólo raramente van a 
causar patologías específicas definidas, pero que pueden llegar a producir disminución de la capacidad funcional del sistema nervioso central, alteraciones del estado psicológico y otros cambios del comportamiento.

De igual forma, otro indicador que se debe controlar para contribuir en la CVL del trabajador es el Medioambiente Tecnológico, esta condición es raramente considerada en la literatura, sin embargo, es un hecho constatable la frustración que genera en el trabajador el no poder disponer de los equipos e instrumentos adecuados para la correcta realización del trabajo que se tiene encomendado, así como la deficiencia en el mantenimiento o en el suministro de componentes. A continuación, se mencionan algunos de los medios tecnológicos, tales como: (Internet, Intranet, Extranet, Correo Electrónico, Videoconferencia, Informática, el Teletrabajo, entre otros.)

En este mismo contexto, se puede decir que el Medioambiente Contractual, es otra condición que suma en la CVL del trabajador, ya que, el salario es uno de los aspectos más valorados del trabajo, aunque el potencial motivador del mismo se haya en función de muchas variables. Un aspecto para tener en cuenta en este sentido es la Teoría de la Equidad de Adams en la que se establece que las personas en situaciones de trabajo distinguen entre contribuciones que ellos aportan y compensaciones obtenidas a cambio, y establecen una razón entre las contribuciones y las compensaciones recibidas y lo que reciben otras personas. De este proceso resulta la percepción de equidad o falta de la misma. La consecuencia de esta última situación es un estado de tensión que impulsa a la persona a intentar reducir ese desequilibrio. Cuando los trabajadores no consideran su salario relacionado con el rendimiento pueden producirse insatisfacción, absentismo, deseo de abandonar la empresa, bajo rendimiento, falta de dedicación, conflicto de intereses, etc.

Por lo cual, la estabilidad del puesto de trabajo supone una relación positiva con la satisfacción laboral y con el compromiso con la organización y presenta relaciones negativas con ansiedad, depresión, irritación, quejas somáticas, úlceras y otras alteraciones. Además, en las situaciones de inestabilidad del puesto de trabajo las personas están más predispuestas a aceptar sobrecarga de trabajo y condiciones ambientales menos adecuadas, que añaden más experiencias estresantes a la ya existente de la inestabilidad. 
En este orden de ideas, otro tópico que contribuye a la CVL del trabajador es el Medioambiente Productivo, del cual se puede exponer que el trabajo por turnos y el nocturno son frecuentes en el medio que nos ocupa. El trabajo por turnos y nocturno supone una gran dificultad en el mantenimiento de una vida familiar y social normal, siendo frecuente que estos trabajadores se quejan de aislamiento social. Además de este problema, es frecuente que los trabajadores presenten problemas de sueño y fatiga y alteraciones en los ritmos circadianos. No parece existir una solución globalmente satisfactoria ya que un mismo sistema de turnos afecta de modo diferente a cada individuo. El grado de estrés e insatisfacción experimentado, que puede llegar a afectar al bienestar y la salud, dependerá de la situación familiar del trabajador (edad de los hijos, aceptación familiar), de su personalidad (introversión-extraversión y neuroticismo), de sus condiciones físicas (edad, ritmos circadianos), de la flexibilidad de sus hábitos de sueño, de su capacidad para superar la somnolencia, etc. Una manera de paliar en parte las consecuencias negativas del trabajo por turno es, además del control de las variables mencionadas anteriormente, permitir la participación de los trabajadores en el diseño de los horarios.

La sobrecarga de trabajo, tanto cuantitativa (exceso de actividades a realizar en un determinado periodo de tiempo o número excesivo de horas de trabajo) como la cualitativa (excesiva demanda en relación con las competencias, conocimientos y habilidades del trabajador o gran nivel de responsabilidad en la toma de decisiones) está asociado al estrés laboral e insatisfacción. Por otra parte, es necesario considerar la otra cara de la moneda: la falta de tareas durante la jornada laboral, así como la asignación de tareas rutinarias y aburridas con relación a las habilidades del trabajador, que también produce insatisfacción.

Otra condición importante en el desarrollo de la CVL del trabajador es el Medioambiente Profesional, con base a un análisis se puede observar que las oportunidades de promoción y ascenso a lo largo de la carrera profesional, así como las facilidades para la formación constituyen importantes factores motivacionales, por cuanto permite un mayor desarrollo de la persona, un aumento de la autonomía laboral y la posibilidad de realizar tareas más interesantes y significativas. Por esta razón, en lo que se refiere a la formación, las organizaciones deben estimular y facilitar las actividades formativas, tanto en el seno de la propia institución como fuera de ella, poniendo a disposición de sus trabajadores los recursos necesarios para la consecución de este fin. 
Las posibilidades de promoción, como ya se ha comentado, constituyen un importante elemento motivador de la actividad profesional. (Pérez, 1999) refiere, la carrera profesional como un sistema de promoción profesional integrado por diferentes categorías a las que se accede consecutivamente, debido a los méritos y actividades desarrollados a lo largo de la vida profesional. Desde esta perspectiva, la carrera profesional es tanto una herramienta de administración de empresas y gestión de RRHH, como la posibilidad de satisfacer las necesidades de promoción de los trabajadores.

Después de este análisis, evidentemente los factores asociados a la dimensión objetivas expuesta anteriormente, impactan de manera importante en la CVL, los cuales percibidos negativamente por parte de los trabajadores (as) pueden llegar a convertirse en causas de procesos estresores en el trabajo, si los mismos no son gestionados adecuadamente y una fuente importante de problemas en la administración del talento humano en las empresas.

\section{Dimensión subjetiva de la calidad de vida laboral}

La calidad de vida es el bienestar, felicidad, satisfacción de la persona que le permite una capacidad de actuación o de funcionar en un momento dado de la vida.

Desde un enfoque subjetivo, propio de cada individuo, se puede mencionar que la calidad de vida laboral está muy influenciada por el entorno en el que vive, como lo representan la sociedad, cultura, escalas de valores, salud percibida, familia, amistades, entorno social, redes sociales, entre otras.

En este sentido, se hace referencia que los factores subjetivos están identificados con las condiciones no medibles (intangibles) del entorno que en sí mismas ayudan a que el trabajador tenga una vida laboral satisfactoria, dichos factores son la retroalimentación en la comunicación descendente y ambos sentidos, la motivación laboral, el mantenimiento de un buen clima organizacional, asociado a las percepciones y valoraciones sobre la mejorabilidad de las condiciones laborales, sobre la influencia a la que está sometido el trabajador y que incide en su estrés laboral, influyen igualmente el entorno social del trabajador como lo son: la familia, los amigos, las redes sociales y culturales, su intimidad. 
En este sentido, las condiciones subjetivas planteadas por Casas y col (2002), abarcan los siguientes aspectos:

- Esfera privada y mundo laboral.

- Interacción afectiva entre lo privado y lo laboral

- Individuo y actividad profesional.

- Satisfacción con el propio trabajo (Autonomía, Implicación, Reputación del puesto)

- Relación con trabajadores.

- Individuo y grupo laboral.

- Plano humano: relaciones del compañerismo y amistad

- Plano técnico: cohesión y apoyo/soporte mutuo.

- Plano organizativo: comunicación horizontal

- Plano jerárquico micro: liderazgo, comunicación ascendente/descendente, organización del trabajo, control, incentivos, reconocimientos.

- Individuo, grupo laboral e institución.

- Apoyo y colaboración entre unidades.

- Institución y función directiva.

- Percepción de estar dirigidos

- Canales de participación en decisiones.

Según la OMS (Organización Mundial de la Salud), la calidad de vida es: "la percepción que un individuo tiene de su lugar en la existencia, en el contexto de la cultura y del sistema de valores en los que vive y en relación con sus objetivos, sus expectativas, sus normas, sus inquietudes. Se trata de un concepto muy amplio que está influido de modo complejo por la salud física del sujeto, su estado psicológico, su nivel de independencia, sus relaciones sociales, así como su relación con los elementos esenciales de su entorno".

La Calidad de Vida Laboral busca armonizar no sólo los aspectos objetivos, sino los subjetivos dentro de las organizaciones, entre los cuales se puede mencionar la familia del trabajador, las 
amistades, su tiempo de ocio y específicamente su tiempo libre que bien podría utilizarlo en prepararse y capacitarse con el firme propósito de contribuir con su desarrollo profesional, de conciliar y a coadyuvar al logro de los objetivos y metas organizacionales de una manera más segura, con el enfoque de salud hacia el personal y con gran expectativa motivacional al rendimiento laboral.

La Calidad de Vida Laboral es multidimensional y multifuncional debido a que está interrelacionada con todos los procesos administrativos (planificación, organización, dirección y control) y operacionales de las empresas, es decir, comprende básicamente dos grandes dimensiones: las que tienen que ver con el entorno en que se realiza el trabajo (objetivo-tangible) y las que se relacionan con la experiencia subjetiva atribuida específicamente a los trabajadores (subjetivo-intangible).

Por ello, la calidad de vida laboral dentro de las empresas posee aspectos subjetivos, tales como: la intimidad, le expresión emocional, la seguridad percibida, la productividad personal y la salud percibida, la capacitación personal y aspectos objetivos donde se ve involucrado el bienestar material, las relaciones armónicas con el ambiente físico y social y con la comunidad, por último, la salud objetivamente percibida.

En lo que respecta a las condiciones subjetivas de la calidad de vida laboral, como elementos intangibles dentro de las organizaciones, y que repercuten en gran medida en el entorno laboral, se debe establecer el manejo desde la supervisión, el equilibrio y la armonía que debe existir entre la esfera privada de los trabajadores y el mundo laboral donde desempeñan sus actividades y tareas diarias.

En este sentido, Segurado y Agulló (2002) mencionan que: "La vida laboral se ve afectada no solamente por factores intrínsecos al entorno de trabajo, sino también por todo lo que acontece en otras áreas extralaborales que conforman la cotidianidad de los individuos (la familia, los amigos, la red de contactos, el ocio y el tiempo libre). Esta interdependencia da lugar a una estrecha vinculación entre lo laboral y lo extralaboral que, además de condicionar la calidad de vida laboral, también determina los modos de vida y el estatus social de los individuos".

Las interrelaciones entre los miembros del equipo de trabajo son factores determinantes en la salud personal y organizacional de las empresas ya que mantienen un clima organizacional 
favorable para el logro de objetivos tanto organizacionales como individuales. Por ello, las oportunidades de relacionarse entre compañeros de trabajo es una variable habitualmente muy considerada; muy por el contrario, la ausencia de contacto con otros trabajadores o la falta de apoyo y cooperación en la ejecución de tareas y actividades diarias puede producir elevados niveles de tensión y estrés entre los miembros de equipos de trabajo.

Desde este punto de vista, se puede inferir que los aspectos subjetivos de la Calidad de Vida Laboral como factores intangibles en las relaciones de los trabajadores debe basarse en la confianza, la buena comunicación, el apoyo mutuo, entre otros, en definitiva, las relaciones personales motivan a mantener un buen clima organizacional en las organizaciones y constituyen un factor muy importante a la hora de alcanzar el éxito en los equipos y grupos de trabajo.

Por otro lado, se observa que cuando las relaciones entre los trabajadores es poca y en algunos casos inexistente, se dificulta la colaboración, la ayuda mutua; y en consecuencia se generan obstrucciones en los procesos de trabajo que las organizaciones necesitarían romper para que fluyan las tareas diarias, la tutoría directa supervisor-supervisado, el aprender a realizar mejor las tareas en el menor tiempo y costo posible, evitarían generar una fuente importante de estrés laboral en los trabajadores.

Así mismo, muchas tareas y actividades en las organizaciones son resueltas en las relaciones entre trabajadores a través de la cooperación, la coordinación, la convergencia de soluciones y toma de decisiones, bien discutiéndolas en los descansos respectivos, en las monitorias supervisor-supervisado, de la buena comunicación y retroalimentación respectiva.

Es así como desde la supervisión se debe ejercer un buen liderazgo donde se deben poseer los conocimientos necesarios de las tareas y actividades, los planes respectivos, los tiempos de entrega; debe ser capaz de fijar objetivos, motivar al personal a alcanzarlos y debe crear un clima de apoyo mutuo de cohesión al objeto de lograr la producción deseada por las organizaciones en el menor tiempo y costo posible.

En este sentido, en muchas organizaciones, los lineamientos y directrices de los patrones se quedan aguas arriba, o llega muy deficiente en la comunicación descendente, produciendo estrés laboral por tal grado de desorientación; por lo cual, ésta comunicación debe ser clara, sencilla y de fácil acceso, a través de instrucciones desde la supervisión para la realización del trabajo 
rutinario con muy buena retroalimentación permitiendo así favorecer a la satisfacción con el trabajo y la identificación de los trabajadores con la organización

Es así como Casas y col (2002), la planificación comporta el establecimiento de objetivos y a decisión sobre las estrategias y las tareas necesarias para alcanzarlos. El establecimiento de objetivos permite delimitar responsabilidades, proporcionar retroalimentación acerca del desempeño, clarificar expectativas, aumentar la autoconfianza de los trabajadores y reducir el estrés.

En este sentido, desde las organizaciones la medición del rendimiento laboral y la incentivación constituyen herramientas fundamentales para la motivación en el trabajo. La adecuada evaluación del rendimiento, mediante la aplicación de criterios lo menos subjetivo posible para todos los empleados, permite detectar las necesidades de capacitación, el desarrollo de las potencialidades y es la base sobre la que descansa una política salarial de reconocimientos e incentivos lo más justa posible.

Es importante destacar que deben existir canales de participación para los empleados que les permita estar integrados no solo a las organizaciones a las cuales pertenecen sino a la empresa en su conjunto, contribuyendo así con estos canales al sentido de pertenencia y la toma de conciencia, lo cual se favorece a incrementar la satisfacción laboral con el trabajo y su bienestar propio en sí mismo.

\section{Relaciones entre los factores objetivos y subjetivos de la calidad de vida laboral}

La búsqueda de una mejor calidad de vida en el trabajo conlleva a equilibrar los intereses de las organizaciones como lo son los objetivos y metas organizacionales con las demandas y las expectativas de sus empleados, los cuales tienen necesidades e intereses particulares que deben verse satisfechos como lo son la satisfacción laboral en sí, el desarrollo personal y profesional, la motivación, capacitación continua y necesaria, el desarrollo de un buen clima organizacional, el llevar satisfactorias relaciones entre sus miembros, con cohesión, cooperación, liderazgo y una buena comunicación.

Todo lo antes desarrollado permite inferir que la fluctuante dinámica de las organizaciones atribuidas al cambio permanente de los mercados como la globalidad, los cambios tecnológicos, 
la variación permanente de los mercados, dependerá de las empresas a través de la supervisión, la cooperación, la comunicación, la motivación a continuar en equilibrio los aspectos objetivos relacionada con la parte tangible o el entorno de trabajo y subjetivos atribuidos a lo intangible que el trabajador valoriza en su experiencia emocional y social para la obtención de una satisfactoria Calidad de Vida Laboral.

En este sentido, luego de la revisión de literatura realizada y como parte de la experiencia de los autores esta discreción, a continuación, se presenta la figura 1 que relaciona los factores tanto objetivos y los subjetivos de la calidad de vida laboral, de manera conjunta.

En la figura se destacan los conceptos de entorno físico, familia, escala de valores, redes sociales y factores culturales como elementos objetivos. Y como elementos subjetivos se presentan la satisfacción persona, la satisfacción laboral, buena comunicación, bienestar material, apoyo y capacitación laboral.

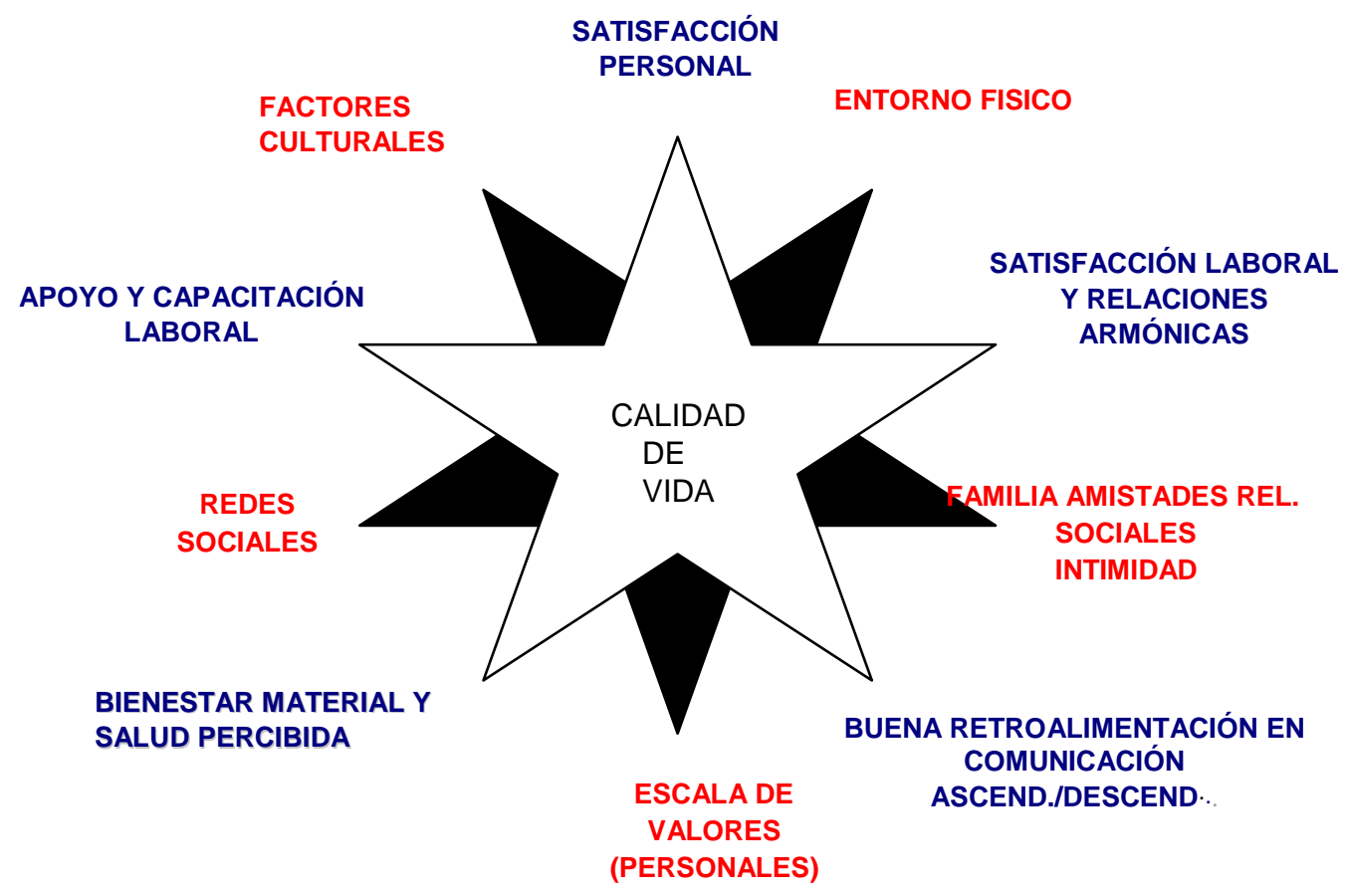

\section{ELEMENTOS OBJETIVOS}

\section{ELEMENTOS SUBJETIVOS}

Figura 1. Relación entre los factores tanto objetivos y los subjetivos de la calidad de vida laboral Fuente: Elaboración propia, con base a Segurado y Agulló (2002) 
El reto de la administración del talento humano, como parte de la gestión empresarial es equilibrar ambas dimensiones de la calidad de vida laboral, para que sirvan como elemento principal para el logro de los objetivos de la organización y también del trabajador de forma armoniosa.

\section{Conclusiones y Recomendaciones}

La calidad de vida laboral es una responsabilidad compartida, es decir, que no solo depende de los trabajadores de la empresa, sino también de los directivos o accionistas de la misma.

No se refiere solamente a la subjetividad de los empleados o de un grupo de estos, ni tampoco se refiere solo a las políticas y prácticas propias del trabajo. Esta se hace realidad en la capacidad que tiene el individuo de afrontar una oposición o de dialogar y negociar con su entorno laboral. Cada persona mejora su desempeño, como resultado de esta interacción y al tornarse más productiva se da lugar a condiciones y recursos que propician entornos laborales de calidad; entonces estos entornos estimulan un trabajo más efectivo.

La calidad de vida laboral puede transformarse en una utopía, porque no existe el medio ambiente laboral ideal. En la medida en que se disfrute del trabajo que se tiene en vez de pretender el trabajo perfecto, se ayuda con la satisfacción y con el manejo del estrés.

Un buen programa de calidad de vida laboral debe incluir los intereses de todas las partes involucradas. No puede hablarse de calidad de vida laboral si los accionistas no alcanzan las metas, o si no se cumple con los requisitos legales aplicables, si no se cuida el medio ambiente social o si los empleados no están satisfechos.

Es necesario tener presente, que la consideración de la calidad de vida laboral por parte de las empresas no tiene fines filantrópicos. Mejorando las condiciones de trabajo se cuenta con talentos más motivados y formados. Sus niveles de productividad aumentan en la misma proporción en que la empresa se interese en las expectativas y necesidades de sus empleados.

La optimización de la calidad de vida sin duda beneficia a los empleados y por ende a la organización. Los empleados se sienten más saludables, reconocidos y retados, trabajan orientados a los objetivos y estrategias del negocio, asumen errores e intentan darles solución, toman riesgos y se comprometen, con la empresa, el entorno y con ellos mismos. 
En este sentido, el concepto de calidad de vida laboral es multidimensional y muy relacionado con las variables que permitirán el éxito en la consecución de objetivos y metas organizacionales como los son: la satisfacción laboral, la motivación, un buen clima organizacional y el satisfactorio rendimiento laboral de los empleados.

Por lo que las organizaciones tendrán que enfocarse en qué aspectos deben ser mejorados, modelar desde la supervisión, identificar y mejorar los problemas identificados, medir y comparar resultados, contabilizar en qué tiempo y qué costos fueron obtenidos y la manera de cómo minimizarlos.

Se recomienda que todos estos aspectos sean planteados en equipos de trabajos para identificar estrategias de mejoras continuada de la calidad de vida laboral, al objeto de permitir identificar cuáles son las causas y consecuencias de los problemas identificados y qué decisiones se deben tomar para orientar sus soluciones.

Asimismo, continuar con una investigación de campo que permita validar en el contexto de la empresa los conceptos desarrollados en este escrito.

\section{Referencias Bibliográficas}

Blanch Josep, Espuny María, Gala Carolina, Martin Antonio. (2003). Teoría de Las Relaciones Laborales. Fundamentos. UOC. España.

Casas, Juana., Repullo, José Ramón, Lorenzo, Susana y Cañas, Juan José. 2002. Dimensiones y medición de la calidad de vida laboral en profesionales sanitarios. Revista de Administración Sanitaria. Vol. 6, N²3: 143-160.

Chiavenato, I. (1999). Administración de personal. Edit. McGraw-Hill, México

Cuesta Gómez, José Luis. (2009). Trastornos del espectro autista y calidad de vida. Editorial La Muralla, S A. Madrid.

Garzón Manuel A. (2005). El desarrollo organizacional y el cambio planeado. Centro Editorial Universidad del Rosario. Colombia.

Jacobs David, Homburguer Alfred. (1992). Como hacer que su empresa sea competitiva. Editorial Díaz de Santos. España. 
López P, Díaz Z, Segredo AM, Pomares Y. (2017). Evaluación de la gestión del talento humano en entorno hospitalario cubano. Revista Cubana de Salud Pública. 2017;43(1): 3-15.

Organización Mundial de la Salud. Programa Envejecimiento y Ciclo Vital. Envejecimiento activo: un marco político. Rev Esp Geriatr Gerontol 2002; 37(S2):74-105

Pérez E, Saavedra Ml. (1999). Motivación y satisfacción. Políticas de incentivación. En: Curso Experto Universitario en Gestión de Unidades Clínicas. Módulo 5. Desarrollo directivo y recursos humanos. UNED, ISCII, ENS.

Schalock, R.L. y Verdugo M.A. (2007). El concepto de calidad de vida en los servicios y apoyos para personas con discapacidad intelectual. Siglo Cero Vol. 38(4). Núm. 224, 21-36.

Segurado Torres, A., \& Agulló Tomás, E. (2002). Calidad de vida laboral: hacia un enfoque integrador desde la Psicología Social. Psicothema, 14 (4), 828-836. 